\title{
Influence of thermal convection on density segregation in a vibrated binary granular system
}

\author{
C. R. K. Windows-Yule, ${ }^{1}$ T. Weinhart, ${ }^{2}$ D. J. Parker, ${ }^{1}$ and A. R. Thornton ${ }^{2}$ \\ ${ }^{1}$ School of Physics and Astronomy, University of Birmingham, Edgbaston, Birmingham B15 2TT, United Kingdom \\ ${ }^{2}$ Multiscale Mechanics, Department of Mechanical Engineering, University of Twente, P.O. Box 217, 7500 AE Enschede, The Netherlands
}

(Received 27 June 2013; published 21 February 2014)

\begin{abstract}
Using a combination of experimental results and discrete particle method simulations, the role of buoyancydriven convection in the segregative behavior of a three-dimensional, binary granular system is investigated. A relationship between convective motion and segregation intensity is presented, and a qualitative explanation for this behavior is proposed. This study also provides an insight into the role of diffusive behavior in the segregation of a granular bed in the convective regime. The results of this work strongly imply the possibility that, for an adequately fluidized granular bed, the degree of segregation may be indirectly controlled through the adjustment of the system's driving parameters, or the dissipative properties of the system's side-boundaries.
\end{abstract}

DOI: 10.1103/PhysRevE.89.022202

PACS number(s): 45.70.-n, 47.55.P-, 83.80.Fg

\section{INTRODUCTION}

Granular materials have been extensively studied for more than two centuries due in part to the plethora of interesting and unusual phenomena they exhibit [1], but also to their relevance in a variety of industrial settings [2] and their importance in geophysical phenomena [3-5]. Granular materials are distinct from classical materials due to the innately dissipative interactions between their constituents [6], giving rise to many of the behaviors observed in granular materials that have no equivalent in classical materials. One such phenomenon is granular segregation, whereby bidisperse or polydisperse granular systems may spontaneously separate into their individual components [7]. Despite extensive research [8], the wide variety of factors influencing the degree to which a system exhibits segregation means that we are still far from fully understanding the phenomenon [9]. Such factors include differences in particle size [10], density [11], and inelasticity [12]; the number of particles in the system and the relative concentration of each particle type [13]; and the strength with which the system is driven [14]. More recently, thermal diffusion has been shown to have a significant impact on segregative phenomena in strongly driven systems [15-17]. However, relatively little experimental work has been performed in this area [18]. One subject which has received relatively little direct attention from the scientific community is the influence of convection on segregation due solely to differences in particles' material properties. "Thermal" granular convection [19] is in many ways analogous to Rayleigh-Bénard convection in classical fluids [20]. For granular systems, where the energy scale of the "normal," thermodynamic temperature is negligible compared to the kinetic energies of the macroscopic particles involved, we define a "granular temperature" $T$ based on the fluctuation of particle velocities about a mean value [21]. For a granular bed excited by, for instance, a vibrating plate at the base of the system, one observes the spontaneous formation of a vertical $T$ gradient due to dissipative interactions within the bed which may, in turn, lead to convective motion. If a granular system is housed in a container with lateral boundaries which are adequately dissipative compared to the bulk of the system, increased relative energy loss at the walls will lead to a locally increased density and decreased $T$ [22], hence, due to buoyancy effects [23], ensuring that flow is always oriented downward at the walls and upward in the center of the system [24], and thus providing a steady state. The aim of this paper is to directly investigate the effect of convection on segregation in a binary granular system whose components are equally sized but differ in their densities and dissipative properties. The results of this work not only highlight the highly significant role of convective motion in the segregation of such mixtures, but also suggest the possibility that the degree to which such a system exhibits mixing or segregation can be controlled by altering the convection strength within the system. It has been shown previously [25] that the strength of the convection discussed above can be tuned through the adjustment of the dissipative properties of the system sidewalls. Hence, one can potentially control the degree of segregation within a granular system simply by altering its wall material, without the necessity of changing the system geometry, the composition of the granular bed, or the method by which it is driven. Clearly, this could prove extremely useful in various industrial applications where segregation may be undesirable, or indeed may be required [26].

\section{EXPERIMENTAL DETAILS}

\section{A. System details}

The main experimental system consists of a granular bed of 500 glass and 500 steel beads, each of $5 \pm 0.1$-mm diameter, housed in a square-based container of height $H=200 \pm 1 \mathrm{~mm}$ and width $W=100 \pm 1 \mathrm{~mm}$. Additional experiments were also conducted using equivalent numbers of glass and brass spheres, as well as monodisperse systems of $N=1000$ glass or steel beads. $H$ is adequate to minimize particle collisions with the upper boundary, and its width and depth are such that the system can be considered fully three dimensional (3D). The relatively large particle size means that interstitial air effects, which may affect segregation phenomena [27], can be neglected [28]. The system walls are interchangeable, allowing the degree of side-boundary dissipation and hence convection within the system to be altered. A complete list of sidewall materials used alongside their respective effective elasticities $\varepsilon_{w}$ can be seen in Table I. $\varepsilon_{w}$ provides a measure of the average change in translational energy during a particle-wall 
TABLE I. Effective elasticities for particle-sidewall collisions for the various wall materials used in the experiment.

\begin{tabular}{lc}
\hline \hline Material & Effective Elasticity $\varepsilon_{w}$ \\
\hline Mild Steel & $0.70 \pm 0.006$ \\
Copper & $0.58 \pm 0.008$ \\
Brass & $0.52 \pm 0.010$ \\
Tufnol & $0.39 \pm 0.012$ \\
Clear Perspex & $0.33 \pm 0.014$ \\
\hline \hline
\end{tabular}

collision due to both normal restitution and rotational motion. $\varepsilon_{w}=1$ corresponds to a perfectly elastic collision, while $\varepsilon_{w}=0$ defines a perfectly dissipative collision. The quoted values correspond to collisions between glass particles and the relevant sidewall material, as measured experimentally by the authors of Ref. [25]. Although values will vary slightly for steel beads, for the purposes of this paper $\varepsilon_{w}$ serves simply as a measure of the relative elasticity of each material, such that general qualitative trends can be observed. The system is vibrated sinusoidally in the vertical direction, imparting energy to the granular bed through particle collisions with the container base. Oscillations of fixed frequency $f=70 \mathrm{~Hz}$ and amplitude $A=1.17 \mathrm{~mm}$ provide a fully fluidized bed. Since vibration is a common method employed in industry for the mixing of granular materials [29], this method of excitation seems appropriate.

\section{B. Positron emission particle tracking}

Data are acquired using positron emission particle tracking (PEPT), a noninvasive technique whereby the time-averaged behavior of a single "tracer" particle in a steady-state granular system can be used to extract information regarding the behavior of the system as a whole. The tracers used are physically identical to the other particles in the system, aside from the fact that they are "labelled" with a positron-emitting radioisotope. The $\beta^{+}$particles emitted from these isotopes rapidly annihilate within the tracer material, causing the emission of a pair of $\gamma$ rays whose trajectories are separated by $180^{\circ}$. Upon placing the tracer between the detectors of a dual-headed $\gamma$ camera, these back-to-back $\gamma$ rays can be used to triangulate the position of the particle multiple times a second, and thus record its motion through a system. Particle motion is recorded in three dimensions with a spatial resolution of up to $1 \mathrm{~mm}$ and a temporal resolution on the millisecond scale [30]. For ergodic, steady state systems such as the one detailed here, the long-time average of a single particle's behavior can be used to extract information pertaining to the system as a whole. For binary and polydisperse systems, individual runs are conducted using tracers of each individual species. The data acquired can then be combined to provide information relevant to the system as a whole. PEPT can be used to determine a multitude of important quantities, including one-, two-, and three-dimensional density and temperature fields $[31,32]$, mean squared displacements and diffusion coefficients [33], convection strengths [24,25], and, in bidisperse and polydisperse systems, individual particle distributions and segregation intensities [34]. Full details regarding the PEPT technique can be found in Refs. [30,33],
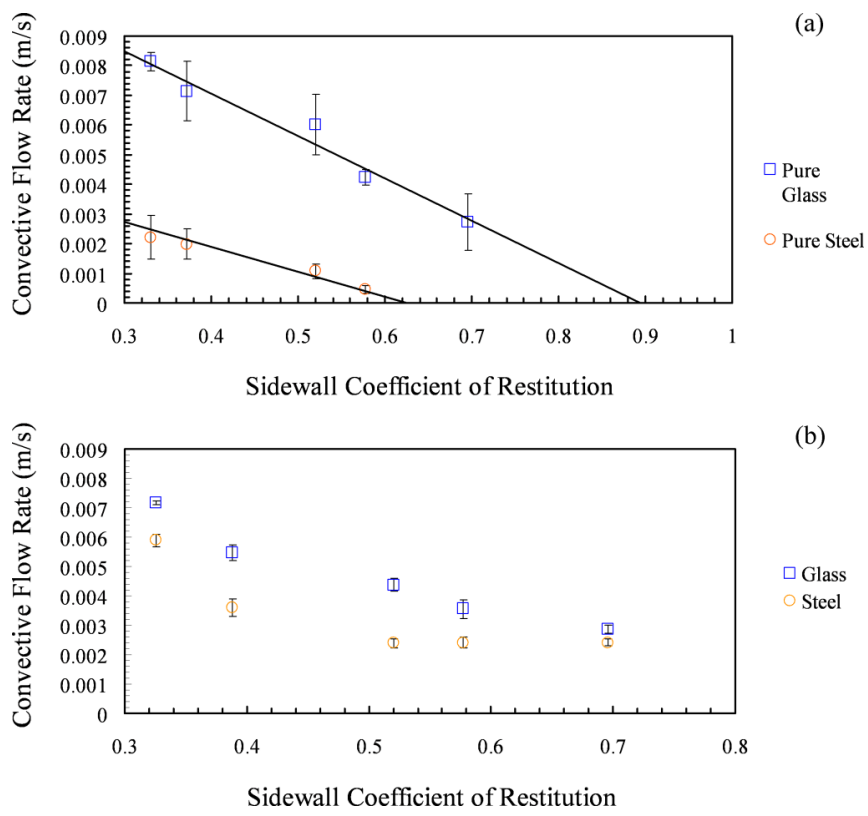

FIG. 1. (Color online) Variation of convective flow rate with $\varepsilon_{w}$ for (a) monodisperse systems of glass and steel spheres and (b) a bidisperse mixture of these materials.

and further information regarding its application to binary systems in Ref. [32].

\section{RESULTS AND DISCUSSION}

Figure 1 shows the variation of convective flow rate $J$ with $\varepsilon_{w}$ for each particle type. $J$ is determined as the average particle velocity through the vertical center of convection for the system, where the horizontal components of velocity are zero [35]. Specifically, $J=\frac{\sum_{i}^{n}\left|v_{z}^{i}\right|}{2 n}$, where $v_{z}^{i}$ is the vertical component of velocity corresponding to the $i$ th location event and $n$ is the total number of relevant data points [36]. Figure 1(a) compares the observed behavior for monodisperse systems of each particle type. In each case $N=1000$. Figure 1(b) shows the behavior for the steel and glass components of a single, bidisperse mixture with 500 of each species, thus keeping the total $N$ fixed at 1000 . The transition from a monodisperse to a binary system shows some interesting effects on the convective behavior of the bed. It is first notable that, for the glass component of the binary system, $J$ is slightly reduced compared to the pure-glass system, whereas for the steel component $J$ becomes considerably larger. This change can perhaps be explained by differences in elasticity between the two components (steel particles are more dissipative than their glass counterparts [37]). Thus the average dissipation in a mixed system will be higher than that of a system composed entirely of glass particles, yet lower than for a pure-steel system, leading to the observed changes in flow rate. This difference in particle elasticity is also believed to explain the considerably reduced $x$ intercept observed for the monodisperse steel system compared to the glass system [25]. The second noteworthy feature is the apparent "plateau" in the convection rate observed in the steel component of the binary system as $\varepsilon_{w}$ increases implying that, unlike the case 
of monodisperse beds, such a system may possess an inherent minimum value of $J$. Further research is required to verify and provide an explanation for this interesting observation. Having discussed the manner in which $\varepsilon_{w}$ affects the convective flow rate of a granular system, we now address the question of how $J$, in turn, influences segregation within said system. The degree to which a binary granular system undergoes segregation or mixing can be quantified in a manner analogous to that found in Ref. [38] by dividing the experimental system into a series of cells and calculating the intensity of segregation as

$$
I_{s}=\left[\frac{\sum_{i=1}^{i=N_{c}}\left(\varphi_{i}-\varphi_{m}\right)^{2}}{N_{c}}\right]^{\frac{1}{2}}
$$

Here, $N_{c}$ is the total number of cells, $\varphi_{i}$ is the single species concentration in the $i$ th cell, and $\varphi_{m}$ is the system's mean concentration. A value $I_{s}=0$ corresponds to a perfectly mixed system while $I_{s}=0.5$ indicates complete segregation. The values of $I_{s}$ discussed in this paper correspond to the equilibrium distribution reached by the system. To confirm that, for all data sets, the system is indeed in a steady state, each 3600 -s run is divided into a series of time intervals. $I_{s}$ is then calculated for each of these windows, and the steady state taken as the point in time at which variations in $I_{s}$ become negligible. In fact, the evolution of the system towards equilibrium is extremely rapid $\left(\Delta t_{\text {seg. }} \ll 3600 \mathrm{~s}\right)$.

Interestingly, $I_{s}$ displays no clear, monotonic dependence on the overall convective flow rate, as might be expected from previous studies $[11,39]$. Neither is there any clear dependence on either of the individual $J$ values for the separate species; rather, it seems that the key parameter is the difference in $J$ between species. Figure 2 shows the variation of $I_{s}$ with the ratio of convective flow rates for the two components of the system, demonstrating the significant degree to which convective behavior can alter the level of segregation.

The role of the differential convection $J_{L} / J_{H}$ between particle species is more clearly illustrated in Fig. 3. The connection between this differential convection and $I_{s}$ can be understood on a qualitative level by considering the fact that denser, and hence heavier, particles are less likely to be dragged into the convective stream [40]. For systems in which convection is weak [Fig. 3(a)], the implicitly small difference in convection rate between the two species leads to a correspondingly small degree of segregation. Conversely, for the case of very strong convective flow, the likelihood of heavy particles being "swept up" in the convective flow increases, leading once again to similar flow rates for each species, and hence a reduced degree of segregation [Fig. 3(b)]. The strongest segregation occurs in the mid-range between these two extremes, where lighter and less dissipative particles undergo significant convective motion, while heavier and less elastic particles remain relatively unperturbed [Fig. 3(c)]. It is interesting to note the stark contrast of these findings to previous studies, which considered only a system's average convection rate and suggested a monotonic relation between this value and the observed degree of segregation. Here, however, we observe the strongest and weakest whole-system convection rates $J_{\text {tot }}$ to demonstrate highly similar degrees of segregation, while the degree of segregation observed for

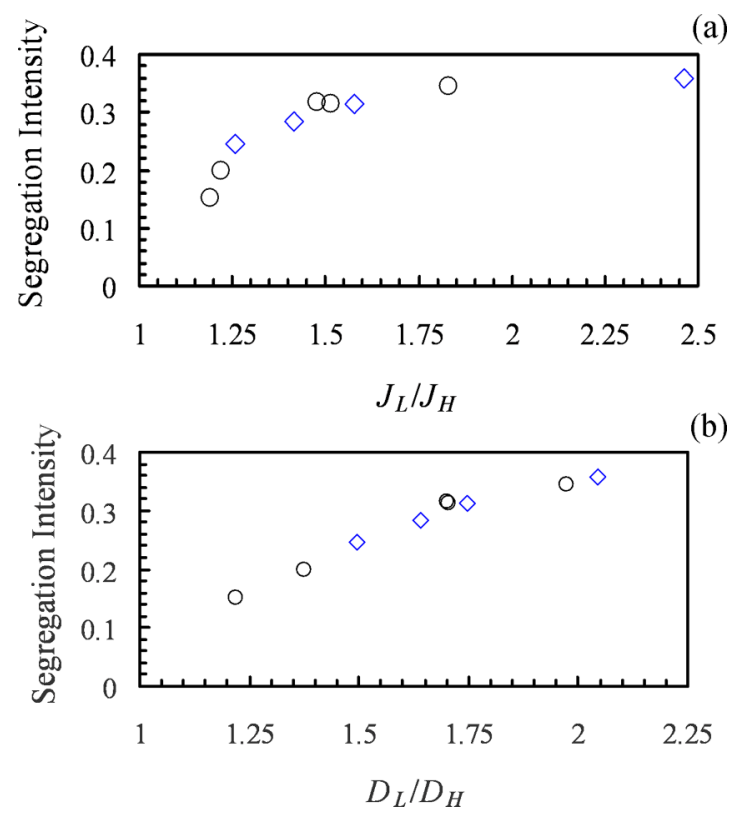

FIG. 2. (Color online) (a) Segregation intensity as a function of the ratio of convective flow rates for light (glass) and heavy (steel) particles. Variation in the value of $J_{L} / J_{H}$ is induced by altering either $\varepsilon_{w}$ (circles) or the system's driving parameters (diamonds). (b) Variation of $I_{s}$ with the ratio of the self-diffusion coefficients $D$ for the two species of particle.

(a)

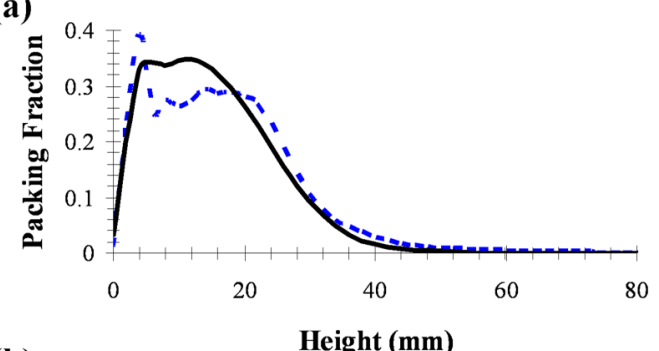

(b)

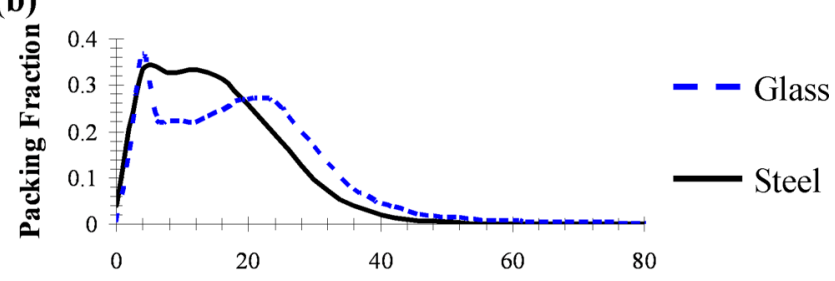

(c)

Height (mm)

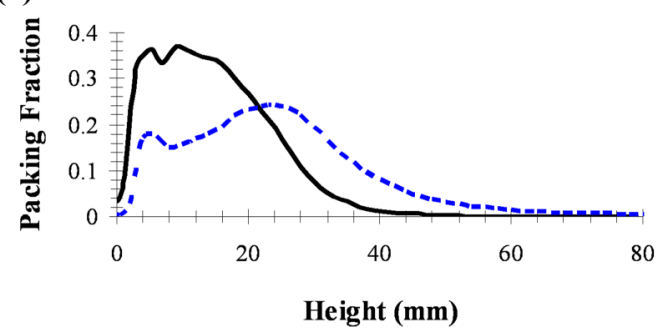

FIG. 3. (Color online) Density profiles for both components of a binary glass-steel system corresponding to (a) steel ( $\left.\varepsilon_{w}=0.70\right)$, (b) perspex $\left(\varepsilon_{w}=0.33\right)$, and (c) brass $\left(\varepsilon_{w}=0.58\right)$ sidewalls. 
intermediate $J_{\text {tot }}$ values can be more than a factor of 2 higher. Such a finding clearly illustrates that future studies of binary and polydisperse systems must more carefully consider the precise behavior of convective flow. Moreover, it is also notable that in the three cases described above, the systems are identical in every aspect other than the material of the walls bounding the system. Thus, Fig. 3 provides a direct demonstration of the ability to control the degree of segregation within a system solely through the alteration of the wall material.

It is perhaps worth noting that, in all cases, segregation occurs predominantly in the vertical direction. However, a small degree of horizontal segregation is also observed, with lighter, less dissipative particles showing a slight tendency to cluster near the walls of the container. This behavior may be simply explained by the greater difference in dissipation between interparticle and particle-wall collisions for more elastic species. The fact that the effect is slightly more pronounced in systems with more dissipative sidewalls lends credence to this hypothesis. Nonetheless, even in the most extreme examples, this horizontal component of $I_{s}$ is an order of magnitude smaller than the vertical, making it a decidedly secondary effect. To verify that convection, and not other sidewall effects, is indeed the dominant factor producing the observed behavior, additional data were obtained keeping $\varepsilon_{w}$ constant and instead altering the convective flow rate of the system by varying the driving parameters. The ranges of driving frequency and amplitude used $[f \in(50,90) \mathrm{Hz}$, $A \in(0.66,1.69) \mathrm{mm}]$ were chosen to ensure variation not only in $f$ and $A$, but also driving velocity $V$ and acceleration $\Gamma$. The collapse of all data points onto a single, monotonically increasing curve suggests that the degree of segregation in the system is not strongly dependent on the driving parameters or specific effects due to sidewall dissipation, supporting the idea that convection is indeed the primary criterion affecting $I_{s}$. Moreover, the lack of correlation observed between $I_{s}$ and the system's density and temperature (which also vary as $V$ and $\varepsilon_{w}$ are altered) implies a lack of dependence on these parameters also. It should be noted, however, that this independence can only be expected to hold in collisionally dominated systems such as the one described here; in higher-density regimes, mechanisms underlying segregation and mixing are markedly different $[41,42]$.

To further investigate the system, additional data were obtained from simulations produced using the MERCURYDPM software developed at the University of Twente [43-45]. The values of $N, f, A, W, H$, and $\varepsilon_{w}$ used in the simulations correspond precisely to experimental values. For intraspecies particle collisions, the values of effective elasticity analogous to the experimental measurements of Feitosa and Menon [37] are implemented; specifically, $\varepsilon_{\text {glass }}=0.83, \varepsilon_{\text {steel }}=0.79$, and $\varepsilon_{\text {brass }}=0.61$. For collisions between dissimilar particles, the effective elasticity is taken as $\varepsilon_{\alpha \beta}=\left(\varepsilon_{\alpha}+\varepsilon_{\beta}\right) / 2$, where $\alpha$ and $\beta$ represent individual species. This relationship arises naturally from the spring-dashpot model of particle elasticity [46-48], and is found to produce good agreement between experimental and simulational results. A collision time $t_{c}=$ $10^{-5}$ is used. The analysis of the evolution of the separate species' vertical centers of mass is used to ensure a steady state. To isolate the effects of inelasticity in the system, the

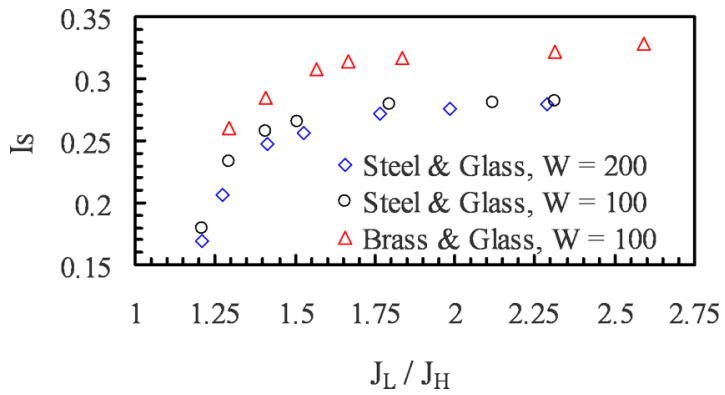

FIG. 4. (Color online) Simulational data showing the variation of segregation intensity with the ratio of convective flow rates of light and heavy particles.

frictional coefficient $\mu$ is set to 0 . As can be seen in Fig. 4, despite some discrepancy in the absolute values of segregation intensity, the general trend of the relationship between $I_{S}$ and $J_{L} / J_{H}$ corresponds closely to that observed in the experiment, demonstrating that the effect of convection persists even in the absence of friction. Moreover, the reintroduction of $\mu$ to the system at values of $0.15,0.5$, and 1.0 was found to produce no significant variation in the results, strongly suggesting that friction does not play a significant role in the observed behavior. This is unsurprising considering the dilute nature of the systems investigated. Simulations were also conducted using the relevant elasticity and density values for a glass-brass system. Once again, the general trend of $I_{S}$ versus $J_{L} / J_{H}$ was found to be consistent. However, certain differences were also observed. First, as can be seen in Fig. 4, the typical magnitude of $I_{s}$ is considerably increased due to the greater disparity in particle masses and elasticities. It was also found that, for a given system, the overall strength of convection was considerably reduced compared to the glass-steel case. This is understandable, as the reduced elasticity of the brass particles will lead to an increased average dissipation for interparticle collisions. This will lead to a reduced density gradient between the central and outer regions of the system, and hence reduced $J_{\text {tot }}$. Conversely, the difference in flow rate between species is typically found to increase. It is worth noting that similar behavior was observed for simulations in which the density ratio was held constant at the steel-glass value and only elasticity was varied. The findings discussed above also agree qualitatively with the experimental observations of brass-glass systems (not shown) [49]. Finally, simulations were conducted using an increased system size. Figure 4 also shows data corresponding to a system for which $W$ has been increased by a factor of 2 and $N$ by a factor of 4 , thus maintaining a consistent resting bed height and ensuring that the control parameter $F_{d}=H(1-\varepsilon)$ [50] is held constant. The closely corresponding behavior of the differently sized systems supports the assumption of a fully 3D domain, as well as providing further evidence that wall effects are not a significant factor in the system's behavior. The slight decrease in the average value of $I_{s}$ for the wider system can perhaps be explained by the decreased relative importance of horizontal segregation. The combination of the above results shows that, despite possible variations in the "baseline" degree of segregation within the system, the ability to significantly vary $I_{s}$ through the alteration of convective 
behaviors applies for a wide range of parameters. Through the use of simulations, it is also possible to investigate more closely the rate at which systems achieve their equilibrium value of $I_{s}$. Despite the significant impact of $J_{L} / J_{H}$ on the magnitude of $I_{s}$, the time $\Delta t_{\text {seg. }}$ in which this segregation is achieved shows no clear dependence on convective behavior; at least, any trends are indistinguishable from the inherent statistical fluctuations in $\Delta t_{\text {seg. }}$ due to the nondeterministic nature of a system's evolution towards segregation. It is, however, interesting to note the rapidity with which the dilute systems investigated here reach their steady state distributions [in all cases, $\Delta t_{\text {seg. }}=\mathcal{O}(10 \mathrm{~s})$ ]. A comparison with the typical timescale of convection $t_{\text {con. }}=\mathcal{O}(1 \mathrm{~s})$, implies that the system reaches its equilibrated state in only a few convective cycles. It is worth noting that the approximate time scale of this surprisingly rapid segregation can be confirmed through visual observation of the experimental system.

Although a full theoretical treatment is beyond the scope of this current paper, we now attempt to provide a qualitative explanation for the variations in segregation strength arising from differences in convective flow. Theoretical work by Garzó $[15,16]$ showed that, in highly fluidized yet nonconvective systems, the degree of segregation reached by a granular bed was determined by a balance between separation caused by thermal diffusion and remixing due to "ordinary" diffusion. In the system currently under investigation, however, one must also consider additional diffusive motion due to convection [51]; the lower probability of heavier particles being dragged into the convective stream [40] will lead to a reduced relative diffusivity for this species. A greater disparity in diffusivity between particle species will give a more significant imbalance between processes favoring segregation and those opposing it. Thus, for the case of highly disparate flow rates between species, their increased relative motion can be expected to create more pronounced concentration gradients and hence increased segregation $[15,16,52]$. Conversely, when convection for both species is equally strong (or, indeed, equally weak), a reduced $I_{S}$ is to be expected. Support for this hypothesis can be seen in Fig. 2(b), which shows a monotonically increasing $I_{S}$ as the self-diffusion coefficients for the two species become more disparate.

\section{CONCLUSION}

Convective motion has been shown to play a crucial role in the segregation of a highly fluidized granular bed whose components differ in their material properties. A mechanism has been proposed to explain a previously unobserved relationship between the convective flow rate and segregation intensity. It is hoped that further research stemming from this observation may lead to a theoretical framework describing segregation in the convective regime. The results of this study strongly imply the possibility that the degree of segregation within a granular bed can be controlled by altering convective behavior through the adjustment of either the driving parameters of the system or the material properties of the container in which it is housed. This latter method may prove particularly useful in situations where it is undesirable or unfeasible to alter the manner in which a system is driven, for example, in certain industrial applications. Moreover, the ability to vary segregation intensity independently of driving force could also potentially lead to significant energy savings.
[1] H. M. Jaeger, S. R. Nagel, and R. P. Behringer, Phys. Today 49(4), 32 (1996).

[2] J. Duran, Sands, Powders, and Grains: An Introduction to the Physics of Granular Materials (Springer-Verlag, New York, 2000).

[3] C. H. Scholz, Nature (London) 391, 37 (1998).

[4] R. M. Iverson, Rev. Geophys. 35, 245 (1997).

[5] P. A. Johnson and X. Jia, Nature (London) 437, 871 (2005).

[6] C.-H. Liu and S. R. Nagel, Phys. Rev. Lett. 68, 2301 (1992).

[7] K. Ahmad and I. J. Smalley, Powder Technol. 8, 69 (1973).

[8] J. M. Ottino and D. V. Khakhar, Annu. Rev. Fluid Mech. 32, 55 (2000).

[9] A. Kudrolli, Rep. Prog. Phys. 67, 209 (2004).

[10] A. Rosato, K. J. Strandburg, F. Prinz, and R. H. Swendsen, Phys. Rev. Lett. 58, 1038 (1987).

[11] S. C. Yang, Powder Technol. 164, 65 (2006).

[12] R. Brito, H. Enriquez, S. Godoy, and R. Soto, Phys. Rev. E 77, 061301 (2008).

[13] N. Burtally, P. J. King, M. R. Swift, and M. Leaper, Granular Matter 5, 57 (2003).

[14] M. Schröter, S. Ulrich, J. Kreft, J. B. Swift, and H. L. Swinney, Phys. Rev. E 74, 011307 (2006).

[15] V. Garzó, Europhys. Lett. 75, 521 (2006).

[16] V. Garzó, New J. Phys. 13, 055020 (2011).
[17] J. J. Brey, N. Khalil, and J. W. Dufty, New J. Phys. 13, 055019 (2011).

[18] K. E. Daniels and M. Schröter, New J. Phys. 15, 035017 (2013).

[19] It should be noted that thermal convection, as discussed in this paper, wherein particle interactions are predominantly inertial, is distinct from the primarily frictionally driven convection observed in less fluidized systems [53].

[20] K. M. Aoki, T. Akiyama, Y. Maki, and T. Watanabe, Phys. Rev. E 54, 874 (1996).

[21] For the sake of brevity, the athermal granular temperature of the system will, for the remainder of this paper, be referred to simply as the temperature.

[22] I. Goldhirsch and G. Zanetti, Phys. Rev. Lett. 70, 1619 (1993).

[23] R. Ramirez, D. Risso, and P. Cordero, Phys. Rev. Lett. 85, 1230 (2000).

[24] R. D. Wildman, J. M. Huntley, and D. J. Parker, Phys. Rev. Lett. 86, 3304 (2001).

[25] C. R. K. Windows-Yule, N. Rivas, and D. J. Parker, Phys. Rev. Lett. 111, 038001 (2013).

[26] A. D. Rosato, D. L. Blackmore, N. Zhang, and Y. Lan, Chem. Eng. Sci. 57, 265 (2002).

[27] X. Yan, Q. Shi, M. Hou, K. Lu, and C. K. Chan, Phys. Rev. Lett. 91, 014302 (2003).

[28] C. Zeilstra, M. A. van der Hoef, and J. A. M. Kuipers, Phys. Rev. E 77, 031309 (2008). 
[29] S. S. Hsiau, C. C. Liao, P. Y. Sheng, and S. C. Tai, Exp. Fluids 51, 795 (2011).

[30] D. J. Parker, R. N. Forster, P. Fowles, and P. S. Takhar, Nucl. Instrum. Methods Phys. Res., Sect. A 477, 540 (2002).

[31] R. D. Wildman, J. M. Huntley, and D. J. Parker, Phys. Rev. E 63, 061311 (2001).

[32] R. D. Wildman and D. J. Parker, Phys. Rev. Lett. 88, 064301 (2002).

[33] R. D. Wildman, J. M. Huntley, J.-P. Hansen, D. J. Parker, and D. A. Allen, Phys. Rev. E 62, 3826 (2000).

[34] C. R. K. Windows-Yule, T. Weinhart, D. J. Parker, and A. Thornton, Phys. Rev. Lett. (to be published).

[35] S. S. Hsiau and C.-H. Chen, Powder Technol. 111, 210 (2000).

[36] For further details regarding the calculation of $J$, please refer to Ref. [25].

[37] K. Feitosa and N. Menon, Phys. Rev. Lett. 88, 198301 (2002).

[38] D. V. Khakhar, J. J. McCarthy, T. Shinbrot, and J. M. Ottino, Phys. Fluids 9, 31 (1997).

[39] H.-Q. Wang, G.-J. Jin, and Y.-Q. Ma, Phys. Rev. E 68, 031301 (2003).

[40] D. A. Huerta and J. C. Ruiz-Suárez, Phys. Rev. Lett. 92, 114301 (2004).

[41] E. Lim, AIChE J. 56, 2588 (2010).

[42] Q. Shi, G. Sun, M. Hou, and K. Lu, Phys. Rev. E 75, 061302 (2007).
[43] A. R. Thornton, T. Weinhart, S. Luding, and O. Bokhove, Int. J. Mod. Phys. C. 23, 1240014 (2012).

[44] A. R. Thornton, T. Weinhart, V. Ogarko, and S. Luding, CMMS 13, 197 (2013).

[45] For a full list of developers, see www.MercuryDPM.org/aboutthe-code/team.

[46] P. A. Cundall and O. D. L. Strack, Geotechnique 29, 47 (1979).

[47] S. Luding, Granular Matter 10, 235 (2008).

[48] T. Weinhart, A. R. Thornton, S. Luding, and O. Bokhove, Granular Matter 14, 531 (2012).

[49] Due to experimental difficulties in creating convection within more internally dissipative systems, only a limited number of experimental data points were obtained. Nonetheless, the differences in the average magnitude of $I_{s}$, as well as the absolute and relative values of $J$ observed in simulation were also present in experimental data. Moreover, the expected monotonic increase of $I_{S}$ with $J_{H} / J_{L}$ was observed, although the number of data points was inadequate to reliably confirm a specific trend.

[50] S. Luding, H. J. Herrmann, and A. Blumen, Phys. Rev. E 50, 3100 (1994).

[51] C. Bizon, M. D. Shattuck, J. R. de Bruyn, J. B. Swift, W. D. McCormick, and H. L. Swinney, J. Stat. Phys. 93, 449 (1998).

[52] D. Serero, I. Goldhirsch, S. H. Noskowicz, and M.-L. Tan, J. Fluid Mech. 554, 237 (2006).

[53] J. B. Knight, Phys. Rev. E 55, 6016 (1997). 